\title{
Mechanism and effects of snow accumulations and controls by lightweight snow fences
}

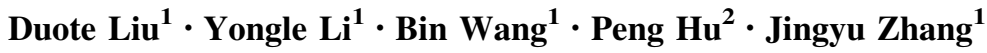

Received: 10 January 2016/Revised: 2 July 2016/Accepted: 5 July 2016/Published online: 23 July 2016

(c) The Author(s) 2016. This article is published with open access at Springerlink.com

\begin{abstract}
This paper investigates the snowdrifts caused by lightweight fences along the lines on the flatland through the computational fluid dynamics method. The characteristic ambient flows around the solid fences and the porous fences with varied heights and bottom wind gaps are simulated in the numerical model, and the working mechanism of "interception" and "scouring" of the lightweight fences are analyzed. Based on the friction velocities near the ground, two sets of criteria are proposed to evaluate the deposition and erosion effects of different fences. According to flow separation and reattachment, the simplified relationships between the most likely positions for snow accumulations and fence parameters are developed. The study indicates that the capabilities for snow interception by the solid fence without wind gap and the distance from which to the second snow coverage center both increase with the fence height. Furthermore, it is found that the scouring range for snow surface increases significantly with the size of wind gap, and the snow accumulation rate on the leeward side decreases with the increasing fence porosity.
\end{abstract}

Keywords Lightweight fence - Snowdrift - CFD . Mechanism of snow accumulation - Fence type $\cdot$ Fence parameter

Yongle Li

lele@swjtu.edu.cn

1 Department of Bridge Engineering, Southwest Jiaotong University, Chengdu 610031, China

2 Department of Bridge Engineering, Changsha University of Science and Technology, Changsha 410076, China

\section{Introduction}

Snowdrifts often occur in snowy alpine regions, which have serious impacts on road conditions and traffic safety $[1,2]$. In addition, long-time snow coverage and snowmelt can accelerate the corrosion of subgrades, pavements, and affiliated facilities [3, 4]. Consequently, maintenance costs will increase [5]. Because the occurrence of snow drifting always comes with airflows, based on the mechanism of particles motion, snow particles are forced to drop to the ground far from the lines through the way of decreasing local wind velocities by continuous members such as early snow walls and snowdrift protection forest $[6,7]$. However, these members mentioned above have to be arranged away from the lines on the windward side, and the uses for snowcontrolling might be limited by terrain conditions.

Other snow protection structures were put forward later. The forms were wind penetrable or bottom-gapped [8-10]. For the differences of the mechanism, the latter should be installed on the road shoulders or bedding slopes near the subgrades. Most of the snow particles floating above the road pavements could be transported further instead of being deposited on the lines by the locally enhanced wind velocities. Compared with those impermeable fences, the parameters of these fences could be more flexible, and the influences on ambient flows and depositions are more complicated.

However, the existing researches aiming at the effects of snow protection by fences with different types and parameters are not adequate. Wang and Chen [8] proposed the schemes of blower fences after studying the energy conversion and flow fields. Besides, based on the mean wind velocities at the height of $0.5 \mathrm{~m}$, the approximate relationships between wind gap sizes and reserved scouring ranges for snow surface were fitted. Zhao et al. [11] 
investigated the general trends of snow cover caused by a single class of fences with only two heights through wind tunnel tests, and the wind direction was set to perpendicular to the line. Nieto et al. [12] simulated the blowing snow disasters on highways and railways with a snow fence via simplified Lagrangian particle tracking model, by ignoring the influences of particle aerodynamic forces and mass transfer effects near the snow surface, and the tracking depended on the spatial mean wind velocities as before. In fact, the mass transfer and deposition of snow particles mainly occur adjacent to the ground. As a result, the analytical methods mentioned above which originated in mean wind velocities far above the ground are not quite appropriate. Accordingly, a method for evaluating the snow protection effects of fences based on friction velocities is proposed in this paper, and the mechanism of snow drift accumulation and the control under different fence types with parameters of fence heights, wind gap sizes, inclinations, and porosities are investigated. The characteristic ambient flows, flow separation, and reattachment phenomenon in snow surface boundary layers are solved using the computational fluid dynamics (CFD) method. In addition, taking predictive variables in snow deposition/erosion model as evaluation criteria, functions of initial snow cover positions versus fence parameters are established. The results can be beneficial to the design and installation of snow fences, wind screens, and noise barriers, even more to the early warning for highway conditions.

\section{Numerical models}

\subsection{Outline of geometric models}

Lightweight fences with heights of $1-5 \mathrm{~m}$ and widths of $0.02-0.04 \mathrm{~m}$ are widely used [13-15], which are considered to be thin-walled structures in contrast to the heavyduty snow walls. They are often arranged in continuous or segmented pattern along the lines where serious snow or sand deposition occurs, following a principle that line and wind direction are orthogonal to prevent disasters $[16,17]$. Due to the differences of actual subgrade sections, the fluctuations of road surface may interfere with the formation of boundary layers to some extent. In order to emphasize the flow fields and snow drifts caused by the fences rather than the topography, an ideal flat land model (Fig. 1) is employed for this study. The wind direction is set to be perpendicular to the fence section and the lane is located on the leeward side. Different fence types and parameters are combined to form different calculation cases as shown in Table 1, including 6 kinds of solid fences with different heights $(H=2-5 \mathrm{~m}), 7$ kinds of solid fences with different inclinations $\left(\alpha=-15^{\circ}\right.$ to $+15^{\circ}$ and $H=3.78 \mathrm{~m}), 6$ kinds of solid fences with different bottom gap sizes $\left(\beta=1 \%-25 \%, H=3.78 \mathrm{~m}\right.$, and $\left.\alpha=0^{\circ}\right)$, and 4 kinds of porous slab fences with different porosities $\left(\eta=35 \%-48 \%, \beta=10 \%, H=3.78 \mathrm{~m}\right.$, and $\alpha=15^{\circ}$.

\subsection{Outline of CFD models}

Fences are located on the open and flat ground. The CFD computational domain and the coordinate system are shown in Fig. 2. The $x$ coordinates for the bottom of all the fences are equal to 0 . Boundary-inlet of the computational domain is "velocity inlet" and the distance to the bottom of the fence is $20 \mathrm{H}$. Boundary-outlet of the computational domain is "pressure outlet" and the distance to the bottom of the fence is $45 \mathrm{H}$. In addition, the boundary-top is symmetrical, and the total height of the domain is $25 \mathrm{H}$. Other boundaries including snow surface and fences are attributed to "nonslip wall." The calculation model is in full-scale. The $k-\varepsilon$ realizable model is used as the turbulence model, where $k$ is turbulent kinetic energy and $\varepsilon$ is dissipation rate of $k$; and the flow fields near the wall surface are solved through scalable function. The minimum size of the mesh depends on the value of local Reynolds number roughly, according to specific cases, which varies in $0.001-0.007 \mathrm{~m}$.

\section{Evaluation methods}

The evaluation methods based on velocities near the ground [8] or spatial mean velocities [12] are only used in preliminary design of subgrade cross sections, and the results are almost crude for the inconsistent reference

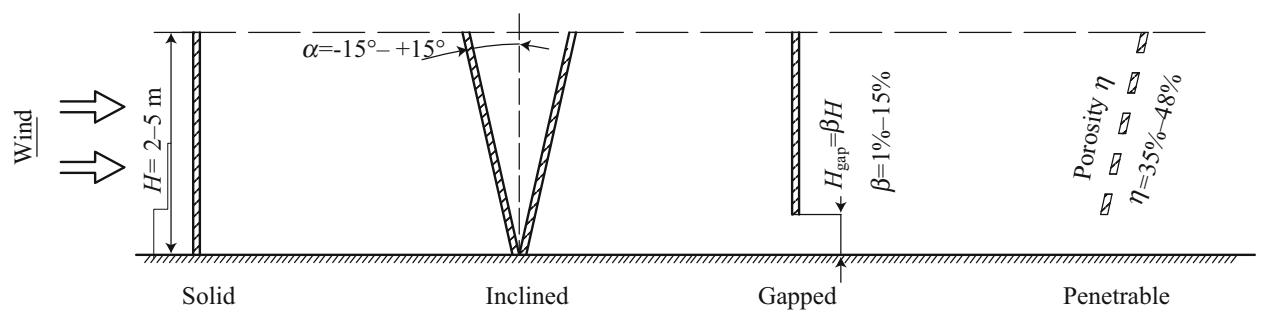

Fig. 1 Fence types and parameters 
Table 1 Cases of combined fence types and parameters

\begin{tabular}{lllll}
\hline Case nos. & Main variables & & \\
\cline { 2 - 5 } & Fence height $H(\mathrm{~m})$ & Inclination $\alpha\left({ }^{\circ}\right)$ & 0 & 0 \\
\hline Case I $(H)$ & $2,2.5,3,3.78,4.4,5$ & 0 & 0 & 0 \\
Case II $(\alpha)$ & 3.78 & $0,5,10,15-15,-10,-5$, & $1,5,10,15,20,25$ & 0 \\
Case III $(\beta)$ & 3.78 & 0 & 10 & $35,39,43,48$ \\
Case IV $(\eta)$ & 3.78 & 15 & & 0 \\
\hline
\end{tabular}

Italics are the main variables in the corresponding case

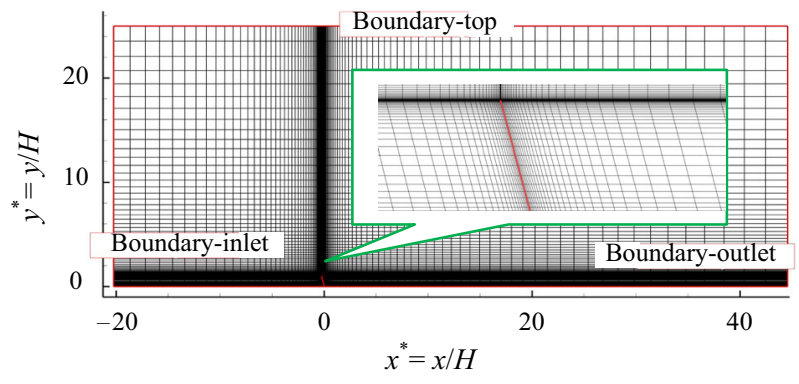

Fig. 2 Computational domain and mesh

standard. Here, an evaluation approach based on a prediction model for snow accumulation is employed [18-20]. Assuming that the breakdown strength of snow surface is bound up with wall shear stress $\tau_{\mathrm{w}}$, the friction velocity $u^{*}=\left(\tau_{\mathrm{w}} / \rho\right)^{0.5}$ near the ground is mainly regarded as the basis to judge whether erosion occurs ( $\rho$ stands for the air density).

Taking into account the relationship between friction velocity inlet $u_{\mathrm{in}}^{*}$ and particle-launch condition $u_{\mathrm{t}}^{*}$ [21], when $u_{\mathrm{in}}^{*} \leq u_{\mathrm{t}}^{*}$ is satisfied, there is no drifting snow from the inlet boundary, and the redistributions of snow deposition caused by ambient flows will never be achieved. Since $u_{\mathrm{t}}^{*}$ changes with weather and snow conditions, the evaluation criterion is difficult to establish. Hence, two criteria for evaluating the possibilities of local deposition are introduced: (1) Relative criterion (RC) $=u^{*} / u_{\text {in }}^{*}=1$, which means if the local friction velocity $u^{*}$ is lower than $u_{\mathrm{in}}^{*}$, snow accumulation will happen; otherwise, the ground is scoured and the lines are in safety. The results are conservative. (2) Absolute criterion (AC) $=u_{\mathrm{t}}^{*} / u_{\mathrm{in}}^{*}$, which means the evaluation depends on both the snow conditions and flow fields. The results are more rigorous.

$u_{\mathrm{in}}^{*}=1.25 u_{\mathrm{t}}^{*}$ is considered to act on the inclined porous fence $\left(\eta=35 \%, \beta=10 \%, H=3.78 \mathrm{~m}\right.$, and $\left.\alpha=15^{\circ}\right)$. The reduced mean velocities at different heights and the contour of mean velocities are shown in Fig. 3. From the way of $[8,12]$, the snow accumulation regions can only be specified roughly for the inconsistent reference standard, and the selections of the measurement point height $z$ have

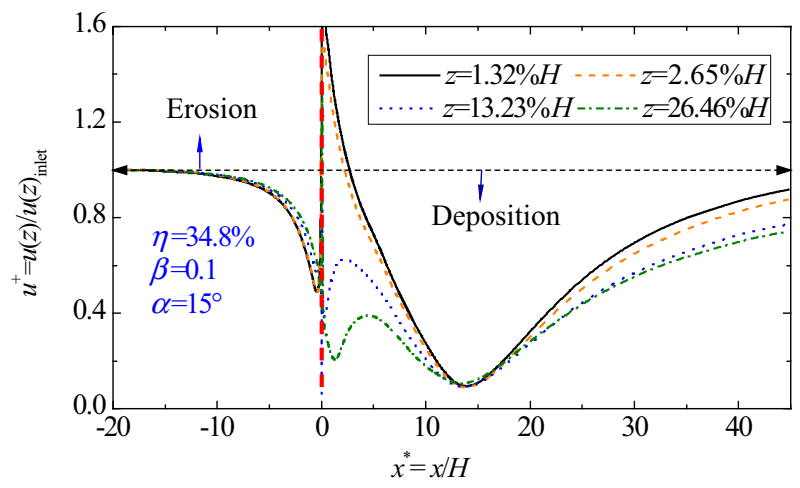

(a) Reduced mean velocities at different heights

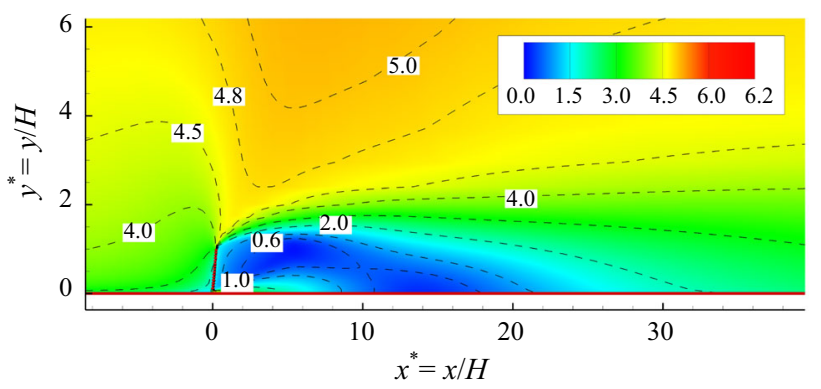

(b) Contour of mean velocities $(\mathrm{m} / \mathrm{s})$

Fig. 3 Calculating mean velocity results

significant influences on the evaluation results. Therefore, a universal conclusion is hard to obtain.

The calculated distributions of dimensionless friction velocities $u^{*+}=u^{*} / u_{\mathrm{in}}^{*}$ near the ground for a solid vertical fence $(H=3.78 \mathrm{~m})$ under conditions of $u_{\mathrm{in}}^{*}=$ $1.0 u_{\mathrm{t}}^{*}, 1.1 u_{\mathrm{t}}^{*}, 1.2 u_{\mathrm{t}}^{*}$, and $1.5 u_{\mathrm{t}}^{*}$ are shown in Fig. 4. There is little difference in the reduced results $u^{*+}$ under different $u_{\mathrm{in}}^{*}$. The two criteria RC and AC are considered together to evaluate the snow protection effects. By RC, evaluation results under different calculation wind velocities are the same. However, higher wind velocities provide a larger scouring area to reduce the possibility of local deposition under AC. 


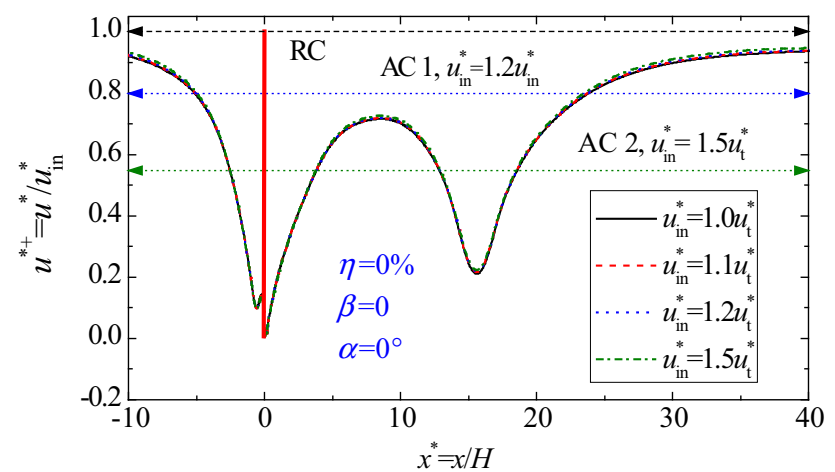

Fig. 4 Two sets of criteria for evaluation

\section{Simulation results and analysis}

\subsection{Fence height}

Solid fences with different heights $\left(H=2-5 \mathrm{~m}, \alpha=0^{\circ}\right.$, $\beta=0 \%, \eta=0 \%)$ are calculated, and the geometrical models used here correspond to Case $\mathrm{I}(H)$ in Table 1 . The inlet wind velocity $u_{\mathrm{in}}^{*}$ is $1.25 u_{\mathrm{t}}^{*}$, and the launch velocity $u_{\mathrm{t}}^{*}=0.15 \mathrm{~m} / \mathrm{s}$ is derived from [21]. The simulated path lines for velocity vectors around the fence are shown in Fig. 5. The dimension of length is reduced to 1 by the height of fence. All calculated results here and above (Fig. 4) show that the changes in $H$ and $u_{\text {in }}^{*}$ make no difference to the features of flow fields. The development of ambient flows can be described as follows: Firstly, the flows rise for the blocking of solid fence, boundary layers are separated near position I at the windward side, and a small vortex $\mathrm{A}$ is formed close to the fence; others get round the fence and a large vortex $\mathrm{B}$ is formed at the leeward side far from the fence. Then, flows reattach to the ground at position II. In this case, local weak shear for ground is generated. Finally, a small vortex $\mathrm{C}$ is separated from vortex $\mathrm{B}$, which is located at position III cling to the fence too. For the velocity decay by eddy dissipation, snow accumulations will occur around positions I-III foremost. Instead, the possibility of snow deposition will be reduced

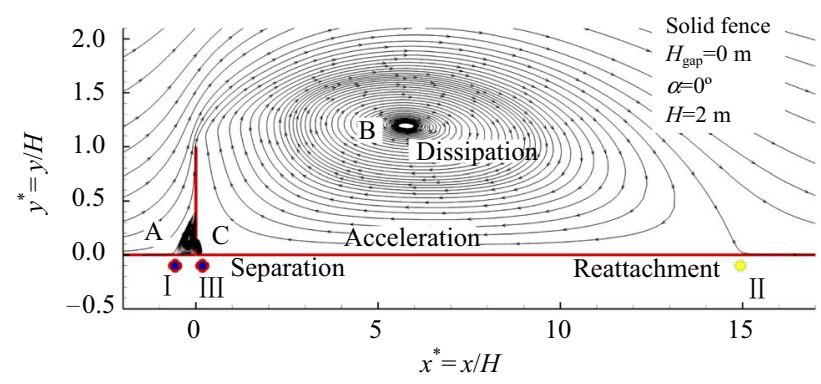

Fig. 5 Ambient flows around solid fence

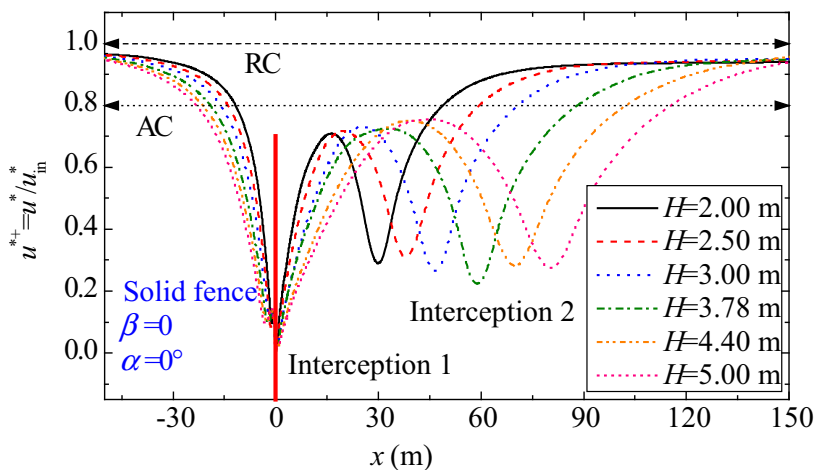

Fig. 6 Performance evaluations for solid fences with varied heights

relatively in the weak shear region where the flows are accelerated during this process.

Friction velocities near the ground under fences with different heights are shown in Fig. 6. The decrement of $u^{*+}$ at the same place close to the fence increases with the fence height, and the same is true for the ranges of deposition. As Fig. 5 shows, the first snow deposition zone mainly appears next to positions I and III, which corresponds to the flow separations and stands for the interception effects. And the second snow deposition zone mainly appears near position II, which corresponds to the flow reattachment. The scale of reverse vortex B on the leeward side increases with the fence height, and the second snow deposition zone moves backwards with position II. Also, we notice that the locally enhanced wall shear stresses for snow erosion behind the fence are scarcely influenced by the fence height under the same inlet velocity. If $\mathrm{AC}$ is used, the state of erosion and deposition may switch due to the value of $u_{\mathrm{t}}^{*}$, and the range of erosion will widen monotonically with an increase in $H$; this effect is also directly related to the change of vortex scale. If $\mathrm{RC}$ is introduced, the reduced friction velocities $u^{*+}$ around fence are always below 1. It means that there is no additional scour generated. In other words, the absolute intercepting effects are represented by this kind of fence. Thus, for solid snow protection structures as snow walls, the higher the fences are, the better the snow protection performances are, but more reserve spaces are needed.

The movements of interception centers with fence heights are shown in Fig. 7. Positions I and III move away from both sides of the fence and there are linear relationships between the fence heights and intercepting positions. As fence height increases, position II tends to move in the same direction as inlet wind, and the relationship between fence height and intercepting position is nonlinear (Eq. 1). According to this, the second snow deposition zone on the leeward side should be apart from the lines in practical applications. 


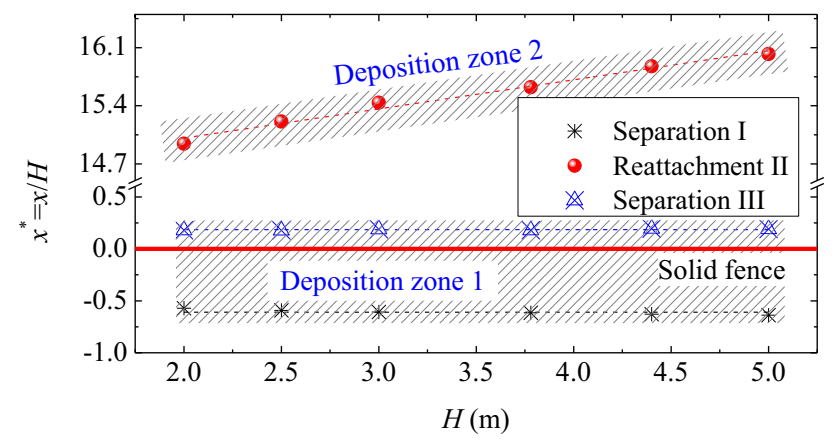

Fig. 7 Positions of interception versus fence heights

$$
\left\{\begin{array}{l}
\text { I: } x=-0.6092 H \\
\text { III: } x=0.1846 H \\
\text { II: } x=14.3098 H+0.3507 H^{2} \\
\left(2 \leq H \leq 5 \mathrm{~m}, \alpha=0^{\circ}, \beta=\eta=0 \%\right) .
\end{array}\right.
$$

\subsection{Inclination}

It is generally accepted that the aerodynamic forces on fence itself are more advantageous when the inclination of fence is in the same direction of wind, but the shearing effects for ground caused by ambient flows still need to be investigated. The relevant parameters of fences are set as follows: the height $H=3.78 \mathrm{~m}$ (Wyoming snow fence), the inclination $\alpha=-15^{\circ}$ to $+15^{\circ}$, as shown in Fig. 1 and Table 1 [Case $\mathrm{II}(\alpha)$ ] above. The positive value of inclination means that the fence inclines to the leeward side of lines, and vice versa. Seven kinds of solid nonblower fences with different inclinations are calculated, and the results are shown in Fig. 8.

Although the range of inclination is $30^{\circ}$ and the offset distance on the top of the fence is nearly $2 \mathrm{~m}$, the differences on friction velocities and flow fields between inclined and vertical fences with the same height are not obvious. As a result, the interception capacity and ranges of deposition in each corresponding region do not change

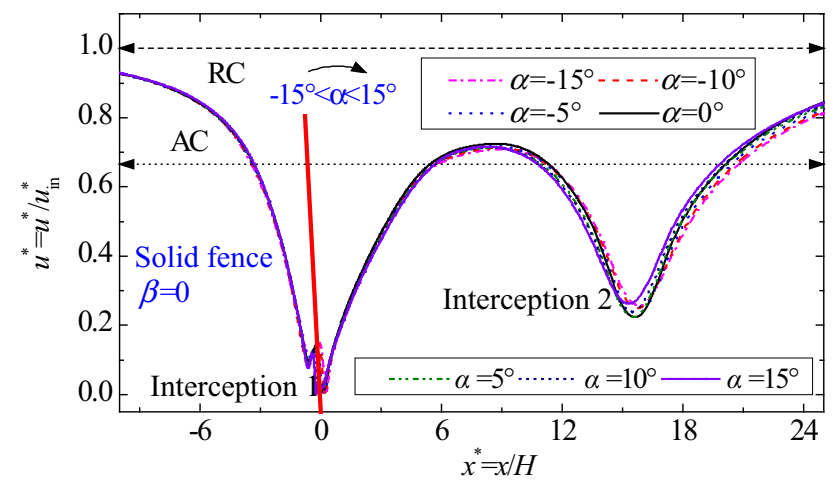

Fig. 8 Performance evaluations for solid fences with varied inclinations

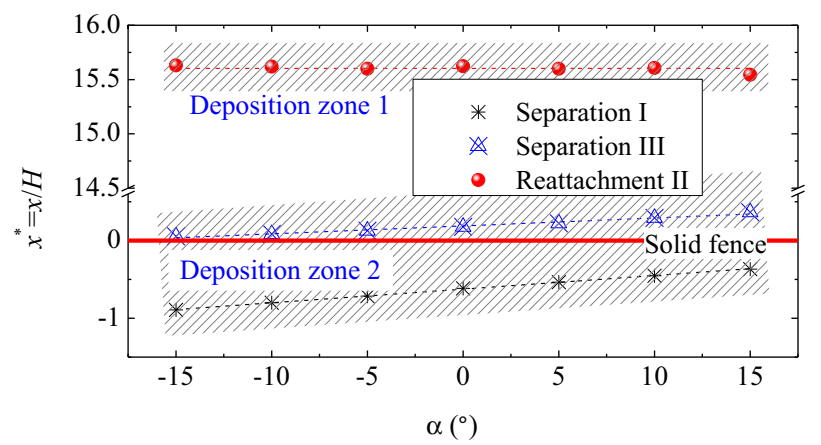

Fig. 9 Positions of interception versus inclinations

with the fence inclinations. Figure 9 shows the movements of interception center with fence inclinations. Compared with the impact of parameter $H$, the moving directions of separation positions I and III are consistent with the swinging directions of inclined fence while the reattachment position II is immobile (Eq. 2).

$$
\left\{\begin{array}{l}
\text { I: } x=-0.6256 H+0.0175 \alpha H, \\
\text { III: } x=0.1891 H+0.0102 \alpha H, \\
\text { II: } x=15.6034 H, \\
\left(15^{\circ} \leq \alpha \leq 15^{\circ}, H=3.78 \mathrm{~m}, \beta=\eta=0 \%\right) .
\end{array}\right.
$$

\subsection{Gap size of blower fence}

Fences will possess the features of "snow dredging" after opening gaps at the bottom of them or lifting them up. The working principle is that flows passing through the wind gaps are accelerated and then act on the ground in a certain distance from the fences. In order to investigate the effects of the gap size alone, vertical solid fences are simulated in this part, and the geometrical models used here correspond to $\operatorname{Case} \operatorname{III}(\beta)$ in Table 1. As shown in Fig. 10, when the size of the bottom gap $H_{\text {gap }}=\beta H$ is set to $1 \% H$, the flow field characteristics change slightly compared with the nonblower fences (Fig. 5). Three vortex areas A-C appear near the fence. Because of the energy supplement by gap flows, the scale of small vortex $\mathrm{C}$ becomes much larger than that of the nonblower fence. The separation positions I and III move forward in the wind direction and the reattachment position II moves backward after vortex B is compressed by the accelerated flows. With the continuous increasing of gap size $\beta$, the scale of vortex $\mathrm{C}$ increases gradually while vortexes $\mathrm{A}$ and $\mathrm{B}$ shrink. When the gap size is $10 \% H$, vortex A disappears and vortex D is generated from the extrusion of vortexes B and C. And the boundary-layer flows pass through the wind gap and separate from the ground at position III only. After the bottom gap rate $\beta$ is over $25 \%$, the vortex B completely disappears and the separation position III merges with the reattachment position II. The ambient flows around fence 


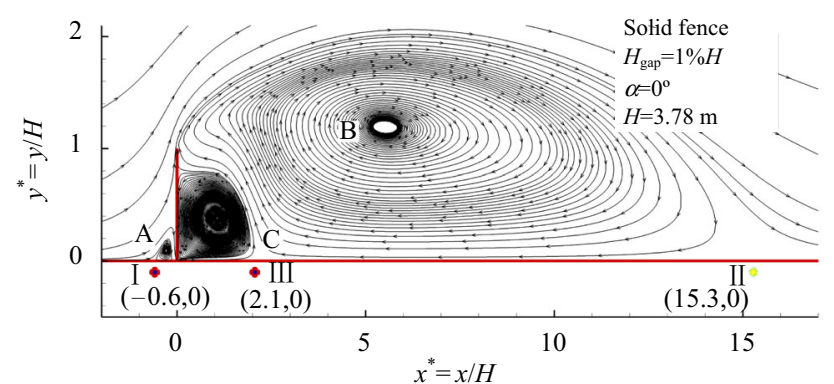

(a) $\beta=0.01$

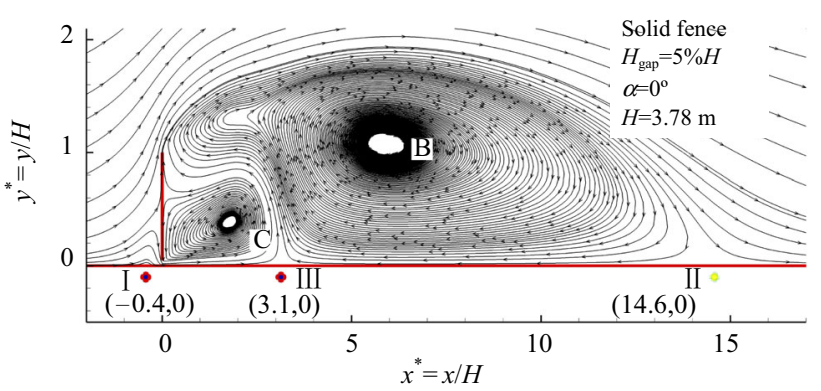

(b) $\beta=0.05$

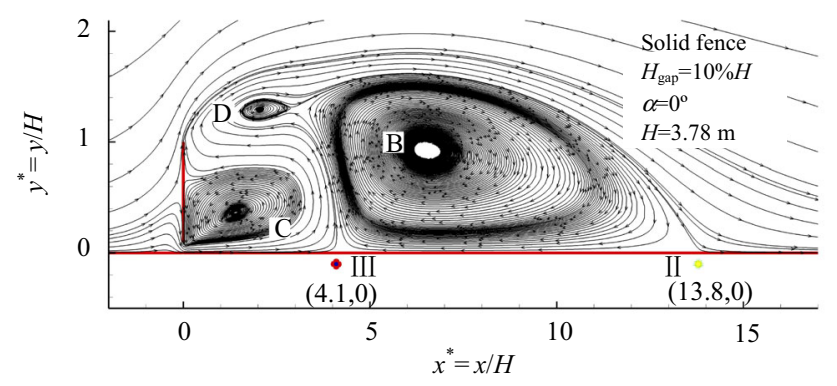

(c) $\beta=0.1$

Fig. 10 Ambient flows around solid fences with different gap sizes

can be deemed as plate flows approximately with $\beta$ over $25 \%$.

The evaluations for blower fences are shown in Fig. 11. Due to a transformation in snow protection mechanism, shearing effects generated on the leeward side of fences are much stronger than near the inlet boundary. Under these conditions, when the RC is used, the bottom gaps on the blower fences provide comparatively abundant security reserves for the nearby areas. Friction velocities behind the fences reach minimal values at positions II and III. Snow deposition at these two positions still have the potential to emerge, and a weaker shear between them caused by the accelerating flows of vortex $B$ is also found. Besides, these average center positions of erosion are almost fixed and in

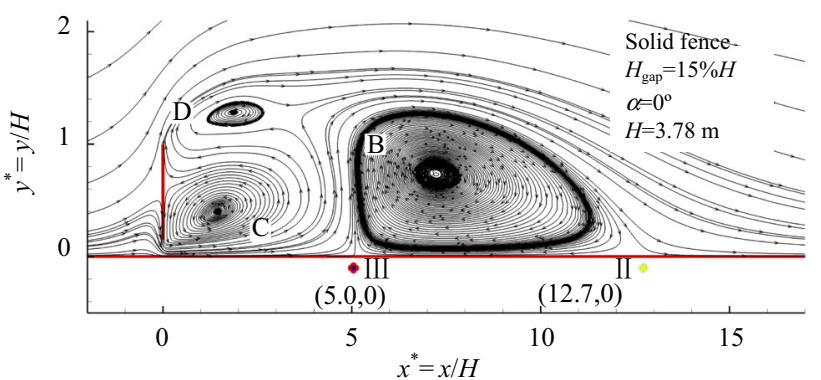

(d) $\beta=0.15$

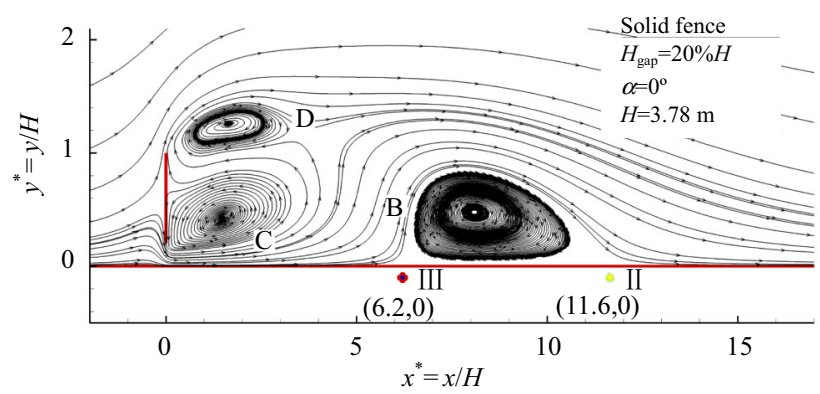

(e) $\beta=0.2$

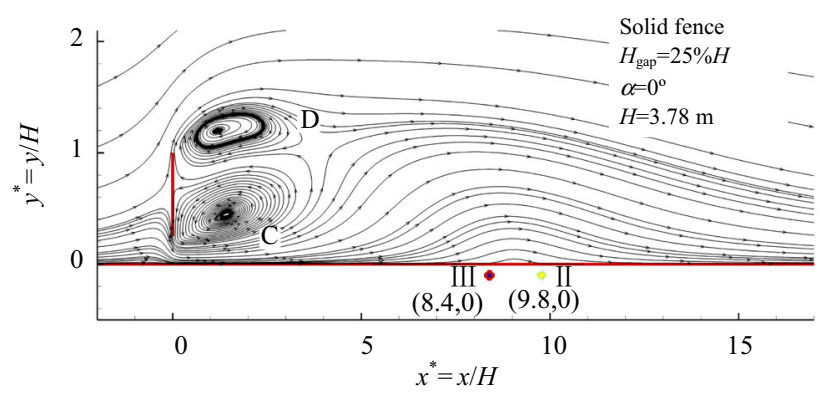

(f) $\beta=0.25$

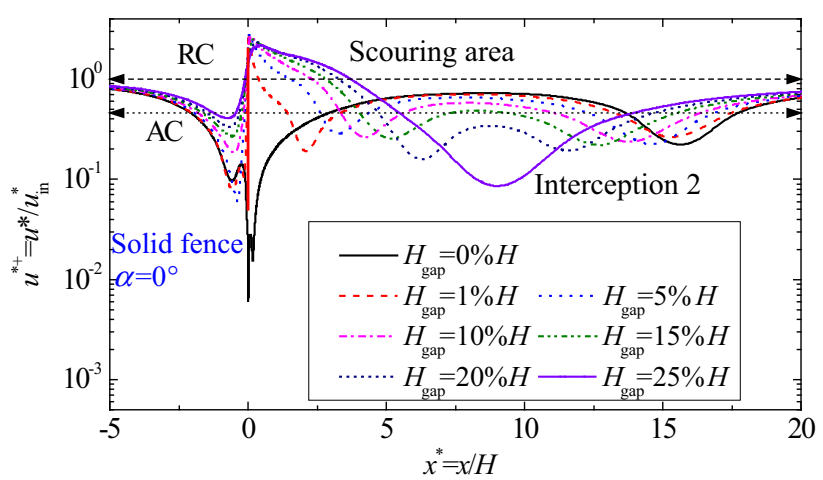

Fig. 11 Performance evaluations for blower fences with varied gap sizes 


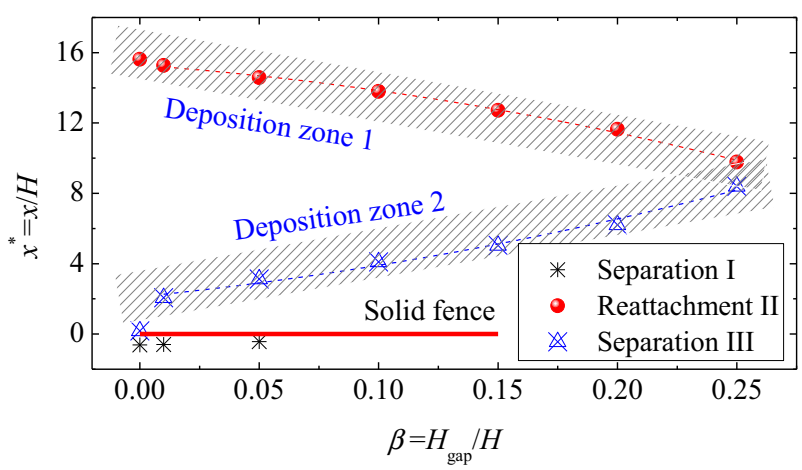

Fig. 12 Positions of interception versus gap sizes

accordance with the one of the solid nonblower fence. With the increase of gap size $\beta H$, the intensity of scour beneath the fence reduces slightly but the scouring range increases significantly. Corresponding to the previous analysis (Fig. 10f), once the gap size $\beta$ exceeds $25 \%$, the local weak shear attributed to vortex B disappears, and the maximum range of scour is about $3.5 H$ (RC: $\beta=25 \%$ ). The local areas in front of fences remain dominated by snow deposition and the ranges of interception do not change with gap size $\beta$.

The movements of interception centers with gap sizes $\beta$ are shown in Fig. 12 and Eq. 3. Combined with Fig. 10, position I disappears after gap size reaches $5 \% \mathrm{H}$, positions II and III move in opposite directions, and the interception regions on the leeward side are gradually close to each other. After gap size is over $25 \% \mathrm{H}$, the boundary-layer flows do not separate from the ground, and the merged snow deposition center is about $10 \mathrm{H}$ from the fence. Thus, without considering the speed of snow accumulation, fences with larger gap size should be used to provide broader scouring scope, and they also need to be installed in proper locations to avoid snow deposition on the lines.

$\left\{\begin{array}{c}\text { II: } x=15.2901 H-9.7696 \beta H-47.4007 \beta^{2} H, \\ \text { III: } x=2.1221 H+13.5925 \beta H+42.6313 \beta^{2} H, \\ \left(1 \leq \beta \leq 25 \%, H=3.78 \mathrm{~m}, \alpha=0^{\circ}, \eta=0 \%\right) .\end{array}\right.$

\subsection{Porosity}

Owing to the ventilation types for noise barriers, wind screens and snow fences varies, and the shapes and directions of holes (or slots) through these structures can affect the flow fields too. In this section, only taking Wyoming snow fences (lath-typed) as research objects, the geometrical parameters are shown in Table 1 [Case $\operatorname{IV}(\eta)]$. All laths and slots of fences are completely identical and arranged at equal interval in order to hold an uniform shielding effect in the active height $(1-\beta) H$. The ambient flows around fences are calculated and shown in Fig. 13. Due to the further reduced blocking areas of porous fences,

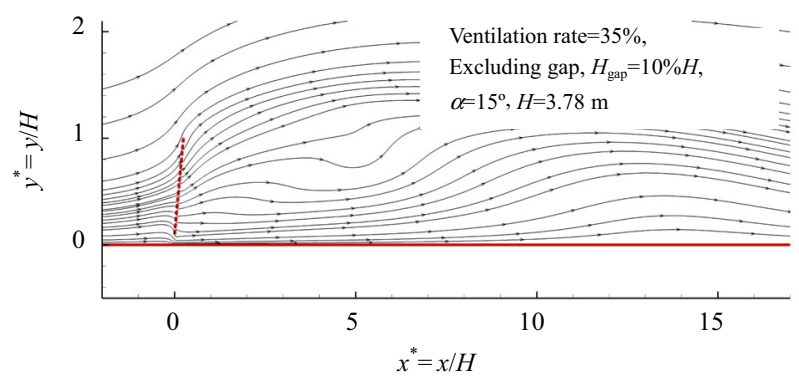

(a) $\eta=35 \%$

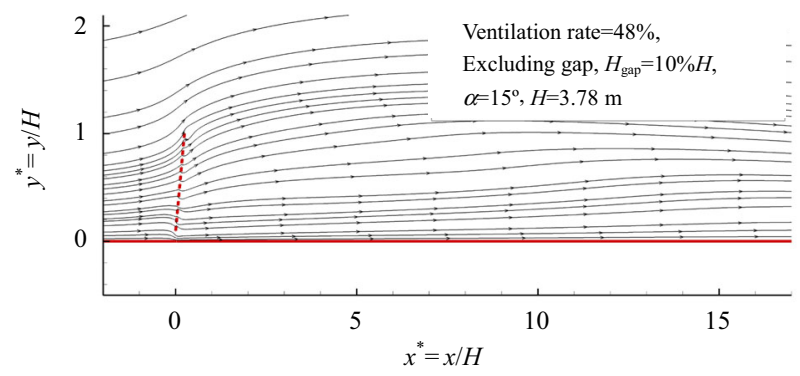

(b) $\eta=48 \%$

Fig. 13 Ambient flows around porous fences with different porosities

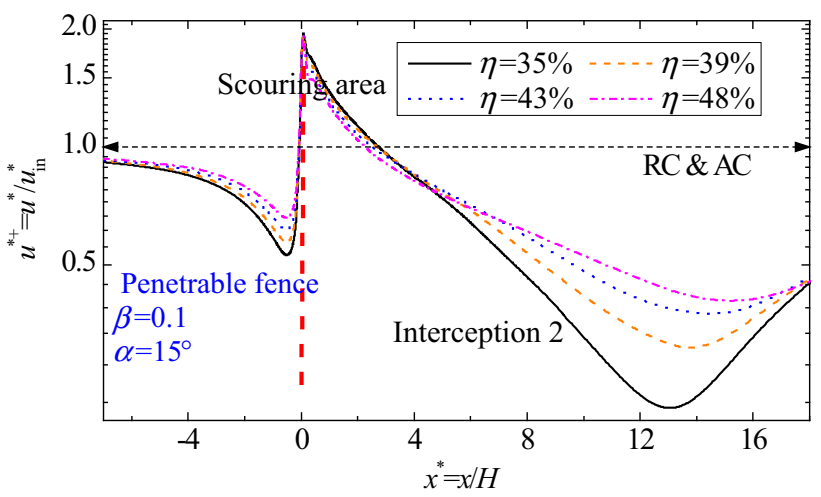

Fig. 14 Performance evaluations for porous fences with varied porosities

boundary-layer separation will no longer appear on the entire ground, and with the increasing porosity, path lines for velocity vectors behind the fences tend to be more horizontally stable. The distributions of friction velocities near the ground under porous fences with four kinds of porosities are shown in Fig. 14. With an increase in porosity $\eta$ from $35 \%$ to $48 \%$, the scouring ranges provided by porous fences are almost the same as those by the solid blower fences with corresponding bottom gap sizes. In addition, the minimal value of friction velocities in the second interception region improves a lot, and the accumulation velocity for snow deposition decreases. Set height $H=3.78 \mathrm{~m}$ and gap size $\beta=10 \%$. By RC, the scouring 
scope on the leeward side is about $2.5 H$, while the one provided by solid fences with the same $H$ and $\beta$ is about $2.3 H$. In summary, when the lath-typed porous fences are used, larger porosity should be prior considered to reduce the snow pack produced in unit time and avoid the snow deposition in the second interception region spreading to the lines ahead of time. The porosity $\eta$ has little to do with the scouring scope which depends more on the size of wind gap $\beta$ and fence height $H$.

\section{Conclusions}

A CFD method for simulating the ambient flows around lightweight fences with varied types and parameters was conducted to investigate the snow protection effects using different evaluation methods, and the following conclusions were obtained:

(1) According to the result of Case I, for solid fence structures like snow walls, higher fences would have better snow protection performances but need more reserve spaces. A notable attention should be paid to the position relationship between the second snow deposition zone and the lines. In this case, lines should be set in the middle of the two interception zones as in Fig. 6, or far away from the second snow deposition zone on the leeward side as terrain condition allows.

(2) According to the result of Case II, minor changes in inclination $\alpha$ of solid fences could rarely affect snow interception. The moving directions of the flow separation positions on the ground are consistent with the swinging directions of the inclined fences. Taking fatigue behavior of fences into consideration, the same direction for fence inclination and inlet wind flows should be adopted.

(3) According to the result of Case III, blower fences with bottom gaps have both capacities of "snow interception" and "snow dredging." The scouring range adjacent to fence on the leeward side increases with the gap size, but has little to do with the range of snow deposition on the windward side. The weak scouring region far from the fence finally disappears after the gap size $\beta$ is more than $25 \%$. Considering the accelerated airflows on the leeward side, fences with larger gap size are recommended under the condition of ensuring vehicle aerodynamic performance.

(4) According to the result of Case IV, the effects of parameter $\eta$ (porosity) on local scour around fences are not so significant as parameter $\beta$ (wind gap size), and the snow accumulation velocities decrease with an increase in $\eta$. In a real-world application, porous fences with large porosities should be applied without sacrificing the "snow dredging" function.

In addition, the analysis results under fence types and parameters in this paper are determined by the initial ambient flow fields, and hence do not indicate the final or maximum snow deposition. It is worth noticing that, however, sending out early warning signal sometimes comes much earlier than traffic interruption. Thus, the evaluation results can be used for preliminary design and planning. More detailed prediction results for nonlinear time-dependent snow accumulations and controls by fences do require wind tunnel tests or more sophisticated numerical simulation techniques.

Acknowledgments The authors are grateful for the supports of the National Natural Science Foundation of China (No. 51525804), the Sichuan Province Youth Science and Technology Innovation Team (No. 2015TD0004), and the Construction Technology Project of China Transport Ministry (No. 2014318800240).

Open Access This article is distributed under the terms of the Creative Commons Attribution 4.0 International License (http:// creativecommons.org/licenses/by/4.0/), which permits unrestricted use, distribution, and reproduction in any medium, provided you give appropriate credit to the original author(s) and the source, provide a link to the Creative Commons license, and indicate if changes were made.

\section{References}

1. Usami S, Kawamoto Y, Honda Y (2002) The effect of the activity area extension by expressway development in case of heavy snow disasters. J Snow Eng 18:22-30 (in Japanese)

2. Liu HH, Lin Y (2005) Change trend and temporal-spatial distribution of snowdrift in china. Arid Zone Res 22(1):125-129 (in Chinese)

3. Osada T, Kobayashi T (1971) Observation on packed snow on roads. J Jpn Soc Snow Ice 33:23-27 (in Japanese)

4. Li YL, Zhao T, Liu DT, Liao HL (2015) Aerodynamic ambient flow around railway wind screens and features of drifting snow. Tiedao Xuebao J China Railw Soc 37(6):119-125 (in Chinese)

5. Noguchi M, Kobayashi H, Kikuchi T, Kawashima K, Iikura S, Fujii T et al (2011) A snow-melting system using water sprinkling on the extended section of the Tohoku Shinkansen Line and its performance confirmation tests. J Snow Eng Jpn 27:204-213 (in Japanese)

6. Anno Y, Konishi T (1981) Modelling the effects of a snowdriftpreventing forest and a snow fence by means of activated clay particles. Cold Reg Sci Technol 5(1):43-58

7. Shimamura M (2009) Snowdrift protection forest on the railway: its history and today's enhancement. J Snow Eng Jpn 25:8-11 (in Japanese)

8. Zhonglong W, Yuan C (1980) Research on prevention of snowdrifts by blower fences. J Glaciol 26(94):435-445

9. Zhonglong W (1982) A study on the movement of snow drift in Tianshan and its control. Acta Geogr Sin 37(1):51-64 (in Chinese) 
10. Sanudo-Fontaned LA, Castro-Fresno D, Coz-Díaz JJD, Rodriguez-Hernandez J (2011) Classification and comparison of snow fences for the protection of transport infrastructures. J Cold Reg Eng 25(4):162-181

11. Zhao G, Zuo H, Zhang H, Hu C, Xu L (2012) Control effect of snow-retaining wall simulated by wind tunnel simulation experiment. J Arid Land Resour Environ 26(4):107-112 (in Chinese)

12. Nieto PJG, Díaz JJDC, Castro-Fresno D, Muñoz FB (2010) Numerical simulation of the performance of a snow fence with airfoil snow plates by FVM. J Comput Appl Math 234(4): $1200-1210$

13. Sakamoto H, Moriya M, Takai K, Obata Y (2001) Development of a new type snow fence with airfoil snow plates to prevent blowing-snow disasters: part 1, evaluation of performance by blowing-snow simulation in a wind tunnel. J Nat Disaster Sci 23:1-11

14. Sakamoto H, Moriya M, Takai K, Obata Y (2001) Development of a new type snow fence with airfoil snow plates to prevent blowing-snow disasters: part 2, characteristics of the aerodynamic fluid forces of airfoil snow plates. J Nat Disaster Sci 23:13-22
15. Perchanok MS (2015) Snow fence operational and material testing. Cold Reg Eng ASCE (1991) 27:53-60

16. Taillandier JM (1984) Self orientable snow fence or sand fence works. EP, EP0060857

17. Walsh NE, Mccabe TR, Welker JM, Parsons AN (1997) Experimental manipulations of snow-depth: effects on nutrient content of caribou forage. Global Chang Biol 3(S1):158-164

18. Uematsu T, Nakata T, Takeuchi K, Arisawa Y, Kaneda Y (1991) Three-dimensional numerical simulation of snowdrift. J Wind Eng Ind Aerodyn 20(1):65-73

19. Naaim M, Naaim-Bouvet F, Martinez H (1998) Numerical simulation of drifting snow: erosion and deposition models. Ann Glaciol 26:191-196

20. Okaze T, Mochida A, Tominaga Y, Ito Y, Yoshino H (2010) Analysis of snowdrift formation mechanism by solving two transport equations of drifting snow densities. In: Proceedings of national symposium on wind engineering, vol 21. Japan Association for Wind Engineering, p 101-106 (in Japanese)

21. Beyers JHM, Sundsbø PA, Harms TM (2004) Numerical simulation of three-dimensional, transient snow drifting around a cube. J Wind Eng Ind Aerodyn 92(9):725-747 